\title{
Where to Share? \\ A Systematic Investigation of Creative Behavior on Online Platforms
}

\author{
Simon M. Ceh, and Mathias Benedek
}

Institute of Psychology, University of Graz, Austria

\begin{abstract}
Digitalization, underpinned by the ongoing pandemic, has transferred many of our everyday activities to online places. In this study, we wanted to find out what online outlets people use to share their creative work and why they do it. We found that most people posted creative work online at least a few times per year. They especially shared creative content related to creative cooking, visual art, and literature but hardly related to performing art. YouTube, Facebook, and Instagram were the three platforms with the highest familiarity and usage rates; among these, YouTube was most strongly used passively (i.e., to view creative content), while Instagram was most strongly used actively (i.e., to post one's own creative content). We could further differentiate plat-
\end{abstract}

forms that were domain-specific (e.g., Stackoverflow for scientific/technological creativity) from platforms that offer a broader variety of creative content (e.g., Reddit, Blogger). The reasoning behind posting one's creative work online resembled a mixture of technological facilitation, alongside heightened accessibility that allows for feedback and bringing pleasure to one's followers and friends. All in all, this study provides a first overview of where and why people share their creative products online, shedding light on timely forms of creative expression.

\section{KEYWORDS:}

creativity; online; Internet; creative content

\section{Article history:}

Received: April 30, 2021

Received in revised from: June 24, 2021

Accepted: June 28, 2021

ISSN 2354-0036

DOI: $10.2478 /$ ctra-2021-0008

\section{Corresponding author at:}

Simon M. Ceh

E-MAIL: simon.ceh@uni-graz.at 


\section{INTRODUCTION}

The Internet eliminates certain temporal and geographical restrictions to creative action (Literat \& Glăveanu, 2016), but it must not be misunderstood as an unorganized space. Instead, online platforms have become the dominant infrastructural and economic social web model (Helmond, 2015) and have changed how we participate in creative communities. Theoretically, they provide an open digital space for people who share particular creative interests and foster collaboration and exchange. Online platforms also enable their users to potentially reach a big audience - which may bring in publicity and economic success in the long run. In fact, it is much easier to hang something on the wall of the Internet compared to hanging it on the wall of a museum. For example, more than 500 hours of videos are uploaded to YouTube every minute (YouTube Official Blog, 2021); in other words, it would take 30.000 hours to watch what is uploaded to YouTube every hour. Across platforms, this results in immeasurable amounts of content that constantly gets created and published online.

The transition of creativity to online environments also benefits consumers of creative content. When following a certain creative interest, one no longer has to wait for the local museum to exhibit, say, long-exposure shots of trams in cities; instead, why not visit one of the photographydedicated online platforms, such as 500px or Flickr - or take a look at the profile of one's favorite long-exposure-shots-of-trams-in-cities photographer on Instagram. The inspiration drawn from these easily accessible photos may, in turn, inspire oneself for the next creative photography adventure. Indeed, because of the participatory nature of most online spaces for creative work (e.g., crowdsourcing; Literat, 2012), the Internet has brought forward an expanded culture of participation that breaks up the distinction between mere production and consumption of creative content (active vs. passive use; Chae, 2018; Literat, 2018; Literat \& Glăveanu, 2018), where producers and consumers merge to form the produser (Bruns, 2008) - a user who finds inspiration in others' creative work, shares their creative work with his/her audience, and posts his/her own creative content online. Accordingly, online platforms can serve as hubs that allow for different aspects of interaction, such as collaboration or feedback.

One evident phenomenon with the rise of creative participation in online spaces is the emergence of new forms of creativity (Literat \& Glăveanu, 2016). As an example, memes - initially defined as cultural units that can both be adapted and spread (Vásquez \&Aslan, 2021) and understood as an application of Darwinian theory of evolution to culture and society (Seiffert-Brockmann et al., 2018) - are typically combinations of images and text, detached from their original context but combined and refined to create humoristic, parodistic or sometimes malevolent creative work. Memes can even gain political traction, leading to real-world consequences (Zittrain, 2014). That said, memes form an Internet-cultural good, and as with most goods, people start to discuss its value, as evidenced by the appearance of a group on Reddit that creatively discusses the value of memes in a way how brokers would discuss the value of stocks (i.e., r/MemeEconomy; Literat \& Berg, 2019). Online platforms may thus foster new ways of creative activity and facilitate the presentation 
of certain kinds of creative work. For example, it seems more straightforward to post digital art, which is typically generated on devices that can also access the Internet, on online platforms rather than printing it and displaying it offline. For creative work that is created offline, online platforms still offer a broad range of tools to transfer them to online products and present them creatively. Digital art is thus not only a domain of creative activity but also a methodology that introduces a "new wave of creative revolution" (Xing, 2018, p. 2). Original recipes can be presented in blog entries via a combination of text and images, while some may choose to create videos of the cooking process and combine them with auditory or textual instructions. Hence, online platforms serve as an easily accessible alternative to classical cooking books or culinary magazines. And while online platforms may limit creative content to visual and auditory modalities, there is no real restriction to the kinds of creative work that can be displayed in such ways. Not only do online platforms remove certain temporal and physical constraints as compared to offline outlets of creativity, but they also enable their users to transfer offline creative work to online spaces. Taken together, online platforms do not only enable users to produce (new kinds of) creative content with digital tools, but also enable the digitalization of offline creative work.

Studies that investigated platform-specific usage motives have shown that creativity can indeed be one such motive that drives online behavior on platforms such as Instagram (e.g., Sheldon \& Bryant, 2016). Independent of the creative domain and the platform, there can indeed be manifold reasons for posting the outcome of creative activities online, just as there are numerous reasons for engaging in everyday creative activities in the first place (Benedek et al., 2020). Simply having more time to do so was the main reason for increased creative activities during a COVID-19-caused lockdown in France (Lopez-Persem et al., n.d.). Online platforms further offer easy ways to interact, especially to indicate appreciation for content and user through likes or karma. These minimal forms of feedback are commonly compiled into dynamic metrics that add an experience of gamification and peer involvement and thereby support extrinsic motivation to online engagement (e.g., Karmawhoring - reward-seeking via obtaining karma-points on Reddit; Richterich, 2014). Heightened exposure to external judgment has cast significant doubt about modern technology's benefits to creative potential (cf. Runco, 2015; detrimental and beneficial consequences of external motivation on creativity are thoroughly discussed in Hennessey, 2019), adding a potentially detrimental facet of social comparison to creative behavior. Unsurprisingly, social media usage is affecting subjective well-being (Verduyn et al., 2017) and happiness (Chae, 2018). One recent study has linked problematic social media use to reduced creative self-belief, suggesting that an individual's inability to manage everyday life situations and social settings promotes excessive social media usage (Kircaburun et al., 2020). The impact of extrinsic factors can be witnessed by how regularly people assess their number of likes and what they are willing to do to secure their favor (Dumas et al., 2017). At the same time, the participatory nature of online platforms enables users to get to know and interact with other similar-minded creatives, allowing a fruitful debate about their work and refining one's skills in the process. However, online platforms must not be mistaken as an egalitarian or fully inclusive venue (de Saint Laurent et al., 2020; Literat 
\& Glăveanu, 2016). Still, the enabling structure of internet platforms, theoretically removing barriers that would otherwise limit the visibility of one's work to a small group of people, may motivate users to post their creative work. The exposure to a huge audience could even attract customers and thus serve as a material motivation (e.g., in a community of artists and designers; Brabham, 2010). According to a study investigating motives for contributing to a crowdsourced children's book project, intrinsic factors such as enjoyment outweighed extrinsic ones (Literat, 2015). Although the potential limitations of online platform usage must not be ignored, there are many good reasons to seek, share, and support creative work online.

Research has taken first steps in investigating creativity at specific online platforms: For example, de Saint Laurent et al. (2020) found that malevolent creative strategies were used to create anti-immigration hashtags on Twitter, while other research groups have scrutinized the use of colors in the creative online platforms Pinterest and Behance (Bakhshi \& Gilbert, 2015; Kim, 2017). Pinterest was shown to attract users both looking for and sharing information regarding creative projects (Mull \& Lee, 2014), and Instagram users stated creative expression as a motive for their use of the platform (Sheldon \& Bryant, 2016). Given the sheer mass and diversity of creative content shared online, we aimed to take stock of the whereabouts of online creativity. To this end, this study assessed the popularity and scope of application of available online platforms for sharing creative work: What kind of creative content do people post online, which platform do they choose for what content, and what motivates them to post their creative work?

\section{METHODS}

Materials, anonymized data, and analysis scripts are provided on the Open Science Framework (https://doi.org/10.17605/OSF.IO/NQ6CS).

\section{Participants}

We used Prolific to recruit a sample of 250 adults (18-60 years), half from the UK and USA, who were native English speakers. The majority of participants were female (59.6\% vs. $40.4 \%)$, and the mean age was 32 years. $(M=32.33, S D=10.14)$. Most participants were either in full-time $(50 \%)$ or part-time (17.2\%) occupation; $25.6 \%$ of all participants were University students. Also, $25.6 \%$ of all participants stated that they are creative professionals and reported engagement in a broad range of creative work (e.g., burlesque dance, knitting, or tattoo design). None of the participants reported literacy difficulties. Participants received financial compensation for completing the survey.

\section{Procedure}

Data were collected via an online survey at LimeSurvey (www.limesurvey.org). The survey was announced on prolific (www.prolific.co). After reading a short description of the study procedure, participants could participate by following a link. The online survey consisted of four sections: First, 
we assessed what kind of creative content participants post online (What I post). Then, they were asked to indicate what online platforms they know and use (Where I post) and to specify the domains they associated with each platform (Where I can find and post creative content). Finally, participants provided their motivation for posting creative content online (Why I post). On average, the total survey took ten minutes.

\section{Materials}

What I post. To understand what kind of creative content gets posted online, we asked participants to indicate their posting frequency regarding their own, self-generated creative content across nine domains. These domains were selected from a previous study investigating motives for creative activities (Benedek et al., 2020) and adapted to better capture forms of creative content in online environments (e.g., memes). For each domain, we asked participants "How often have you engaged in posting creative content online in your leisure time within the last 12 months?", and to indicate their posting activity for each domain on a 5-point scale: " 0 - never", "1 - sometimes (a few times per year)", "2 - regularly (about once a month)", "3 - frequently (about once a week)", "4 - very frequently (nearly every day)". A description of the domains (with examples of creative content for each domain) can be found in Table 1. In an open follow-up question, participants then described the specific kinds of creative content they typically posted online (e.g., "photos of handmade quilts").

Table 1. Creative domains (adapted from Benedek et al., 2020)

\section{How often have you engaged in posting creative content online in your leisure time within the last 12 months?}

Literature (e.g., posting your own blog entries, texts, poems, jokes)

Music (e.g., posting self-created or self-adapted melodies)

Handicraft (e.g., posting selfmade cards, cloths, bags)

Interior and garden design (e.g., posting your own design/decoration ideas for one's living space)

Creative cooking (e.g., posting self-created dishes/drinks)

Visual art (e.g., posting your own drawings, creative photography/video/film)

Performing art (e.g., posting self-made plays, choreographies)

Science/Technology (e.g., posting your own solutions to technical problems, scientific papers)

Social (e.g., creating your own memes, inventing games)

Where I post. Based on a thorough web search, we identified 29 international online platforms that are commonly used for posting creative content. These included platforms focused on specific creative domains (e.g., photography: Flickr) but also popular social media sites that are commonly used to share creative work. For each platform, participants were asked to indicate whether they know the platform and whether they use it to view creative content from others, to share such content from others, and/or to post their own creative content. In a follow-up question, participants could indicate additional platforms they use in one of the previously mentioned ways. 
Where I can find and post creative content. To get a deeper understanding of what creative domains are associated with certain platforms, we asked participants to associate each of the 29 platforms to the creative domains (Table 1) that best represented the type of content that is typically posted or showcased there (e.g., when using Pinterest for posting recipes and finding new ideas for decorating one's living room, participants would then tick both "creative cooking", and "interior and garden design"). In case participants were not familiar with a platform or did not know which creative domain(s) to associate it with, they could choose "don't know." For analyses of each platform (usage and related domains), we only included participants who stated being platformusers (in at least one of the three previously mentioned ways).

Why I post. We devised twelve statements to assess important motives for posting creative work online, based on a thorough investigation of the theoretical and empirical literature on online creativity (Table 2). The statements were presented in randomized order. Participants were asked to move a slider to indicate the extent of (dis)agreement to each of the twelve statements $(0=$ total disagreement, 1 = total agreement). In an open question, participants could then name additional motives that explain their reasons for posting their own creative content online.

Table 2. Motives for sharing one's creative content online

Why do you post your own creative content online?

I can help to inspire others.

My work might bring pleasure to others.

I can post my work anywhere and at any time.

I can easily showcase my work.

I want to see whether my creative work is of value to others.

I want to see how much positive feedback I get.

It allows me to express my thoughts and feelings.

It allows me to expose my creative vein.

The feedback on my work allows me to refine my skills.

I want to engage with others in discussing my work.

I can earn money with it.

I want to reach as many people as possible.

\section{RESULTS}

\section{What creative content do people post online?}

Most participants (91.6\%) reported to at least sometimes post own creative content online across all domains. However, online posting activity differed considerably across creative domains: Participants posted most frequently in the creative domains of creative cooking, visual art, and 
literature, while they posted only infrequently in the domains of music, science/technology, and performing art, respectively (see Figure 1).
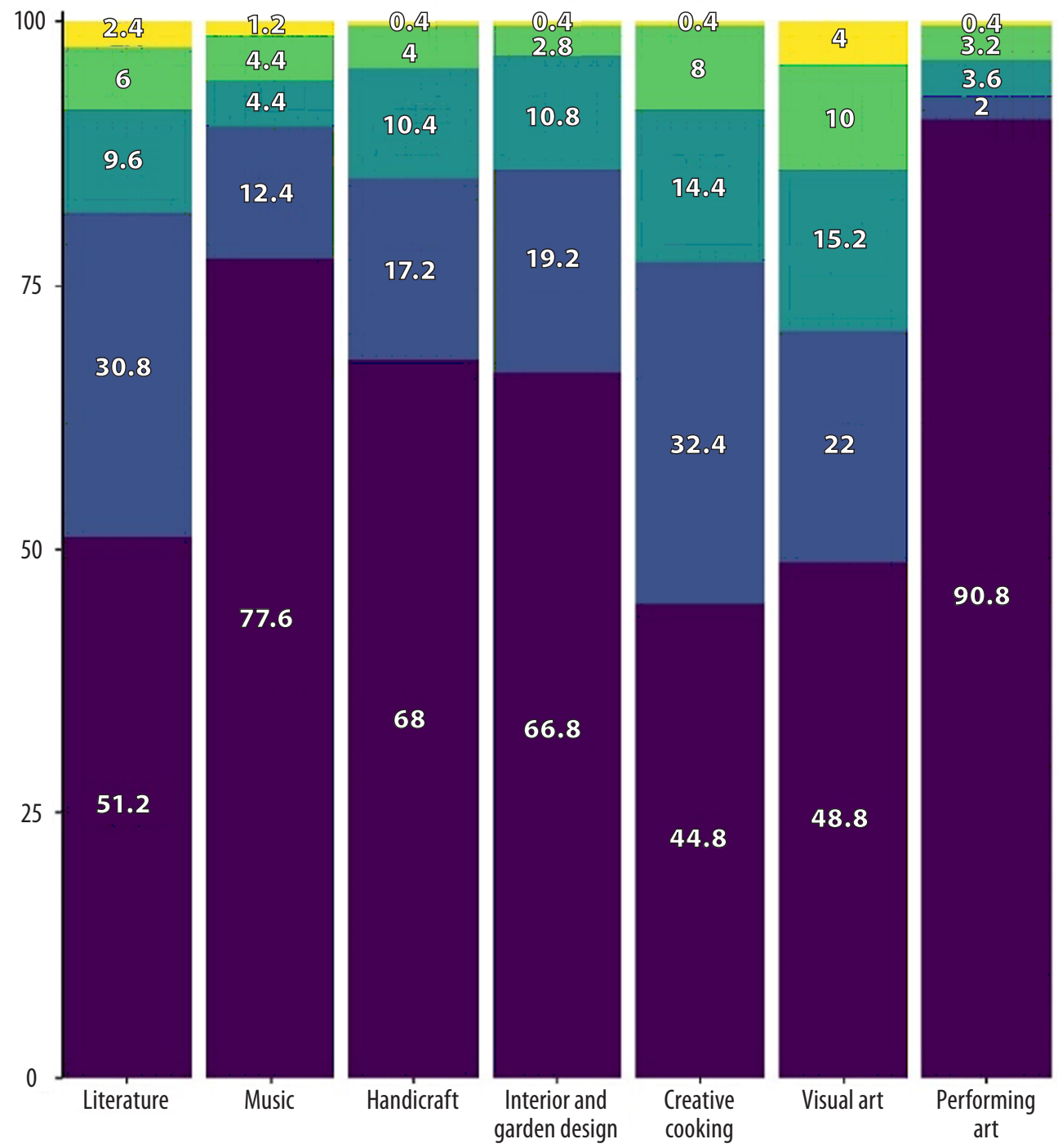

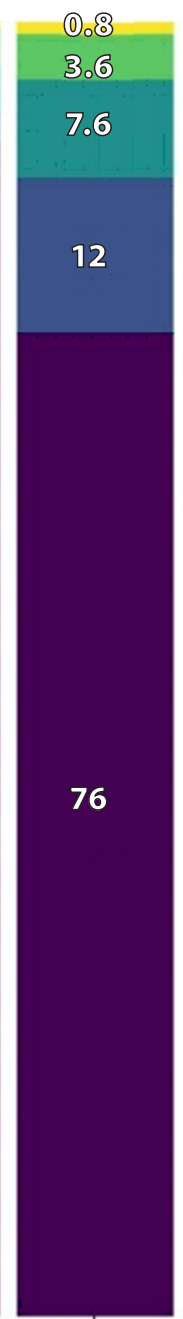

Science/ Technology

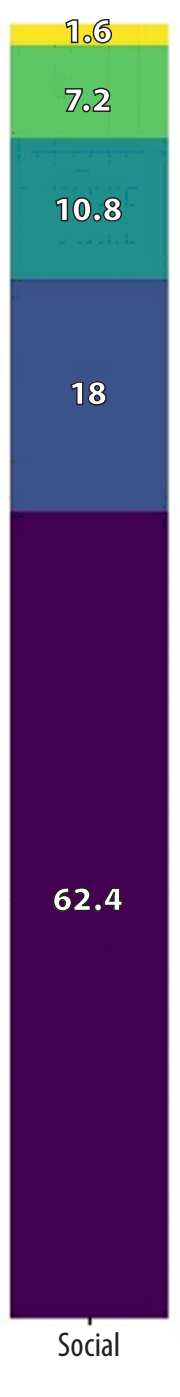

\footnotetext{
Posting activity (\%)

very frequently (nearly everyday)

frequently (about once a week)

regularly (about once a month)

sometimes (a few times per year)

never
}

Figure 1. Frequency of posting activity across nine creative domains

\section{Where do people post creative products online?}

15 of 29 platforms were known to at least $75 \%$ of all participants, while only five platforms were known to less than $25 \%$ (see Figure 2). Seven platforms were used by more than half of the sample: YouTube (84.8\%), Facebook (77.6\%), Instagram (73.2\%), Pinterest (64.8\%), Reddit (59.2\%), Twitter (59.2\%), and Etsy (54.4\%). Participants named 31 additional platforms, four of which were mentioned more than three times ( $>1 \%$ of the sample), including Discord $(n=5)$, Thingiverse $(n=4)$, WhatsApp $(n=3)$, and Archive of Our Own ( $\mathrm{n}=3$; for a complete list, see Table S1 in the supplementary material). 


\begin{tabular}{|c|c|c|c|c|c|}
\hline & Familiarity & User & Use to view & Use to share & Use to post \\
\hline YouTube - & $99.6 \%$ & $84.8 \%$ & $70.4 \%$ & $25.6 \%$ & $18.4 \%$ \\
\hline Facebook - & $99.6 \%$ & $77.6 \%$ & $50.8 \%$ & $33.2 \%$ & $44.4 \%$ \\
\hline Pinterest - & $98.4 \%$ & $64.8 \%$ & $50.8 \%$ & $24 \%$ & $13.2 \%$ \\
\hline Instagram - & $98 \%$ & $73.2 \%$ & $44.8 \%$ & $32.4 \%$ & $51.6 \%$ \\
\hline Twitter - & $98 \%$ & $59.2 \%$ & $45.2 \%$ & $26.8 \%$ & $24.4 \%$ \\
\hline Snapchat - & $97.2 \%$ & $40.4 \%$ & $26.8 \%$ & $10.4 \%$ & $22.8 \%$ \\
\hline Tiktok - & $95.6 \%$ & $40.4 \%$ & $31.6 \%$ & $12.8 \%$ & $13.2 \%$ \\
\hline Reddit - & $94.4 \%$ & $59.2 \%$ & $45.6 \%$ & $22.4 \%$ & $26.4 \%$ \\
\hline Etsy - & $94 \%$ & $54.4 \%$ & $46 \%$ & $5.2 \%$ & $8 \%$ \\
\hline Linkedln - & $92.8 \%$ & $44 \%$ & $33.2 \%$ & $12.8 \%$ & $13.6 \%$ \\
\hline Tumblr - & $90.4 \%$ & $24.4 \%$ & $20.8 \%$ & $8 \%$ & $6.4 \%$ \\
\hline Flickr - & $82 \%$ & $17.6 \%$ & $15.2 \%$ & $2 \%$ & $2.8 \%$ \\
\hline Soundcloud - & $80.4 \%$ & $27.6 \%$ & $21.6 \%$ & $3.6 \%$ & $5.6 \%$ \\
\hline Twitch - & $76 \%$ & $27.2 \%$ & $23.6 \%$ & $5.6 \%$ & $4 \%$ \\
\hline Vimeo- & $75.6 \%$ & $18.8 \%$ & $15.6 \%$ & $2.8 \%$ & $4 \%$ \\
\hline Imgur - & $62.4 \%$ & $24.4 \%$ & $20.8 \%$ & $4 \%$ & $6.8 \%$ \\
\hline DeviantArt - & $53.2 \%$ & $17.6 \%$ & $16 \%$ & $2.8 \%$ & $3.6 \%$ \\
\hline Blogger - & $48 \%$ & $12 \%$ & $8 \%$ & $2 \%$ & $4 \%$ \\
\hline Github- & $47.6 \%$ & $15.6 \%$ & $14 \%$ & $4 \%$ & $4.8 \%$ \\
\hline Wattpad - & $40 \%$ & $7.2 \%$ & $5.6 \%$ & $2.4 \%$ & $0.4 \%$ \\
\hline 9gag - & $37.6 \%$ & $3.2 \%$ & $3.2 \%$ & $0 \%$ & $0 \%$ \\
\hline Stackoverflow - & $33.6 \%$ & $13.2 \%$ & $12 \%$ & $2 \%$ & $1.2 \%$ \\
\hline Fanfiction(.net) - & $32.8 \%$ & $11.2 \%$ & $10 \%$ & $1.2 \%$ & $1.2 \%$ \\
\hline Strava- & $32 \%$ & $8.8 \%$ & $5.6 \%$ & $1.6 \%$ & $4.8 \%$ \\
\hline Researchgate - & $22.8 \%$ & $10 \%$ & $9.6 \%$ & $1.6 \%$ & $0.8 \%$ \\
\hline Behance - & $12.4 \%$ & $4.4 \%$ & $2.4 \%$ & $2 \%$ & $0.4 \%$ \\
\hline Exposure - & $12.4 \%$ & $2.8 \%$ & $1.2 \%$ & $1.2 \%$ & $0.4 \%$ \\
\hline $500 p x-$ & $6.8 \%$ & $1.6 \%$ & $0.4 \%$ & $1.2 \%$ & $0.4 \%$ \\
\hline Commaful - & $6.4 \%$ & $2 \%$ & $1.2 \%$ & $0.8 \%$ & $0 \%$ \\
\hline
\end{tabular}

\section{Figure 2. Platform familiarity and usage}

Note. Percentage scores are relative to the full sample $(\mathrm{N}=250)$. Participants could select up to all three usage patterns (e.g., use to view + use to share).

In a next step, we examined to what extent these platforms feature content from specific creative domains (Figure 3). Closer inspection of the data revealed two patterns: Some platforms were clearly domain-specific, as indicated by high relevance rates in one single domain. For example, Stackoverflow (81.8\%), Researchgate (80\%), and Github (74.4\%) were almost exclusively associated with scientific/technological creativity. Similarly, Soundcloud (87\%) and DeviantArt (84.1\%) were associated with musical creativity, and visual art, respectively. In contrast, other online platforms were found to be more domain-general: they often showed a focus on one or two creative domains, but other domains were also represented to a substantial degree. To give an example, Twitter was strongly associated with social creativity (87.2\%) but also with all other creative domains 
(> 29.7\%). The same holds true for Reddit, a platform associated with social creativity (81.8\%) but also substantially linked to all other creative domains (all $>48.6 \%$ ). Albeit less popular in general, Blogger was the most balanced platform (every creative domain within the range of $26.7 \%-56.7 \%$ ), suggesting that this blogging site is not clearly attributed to any particular creative domain.

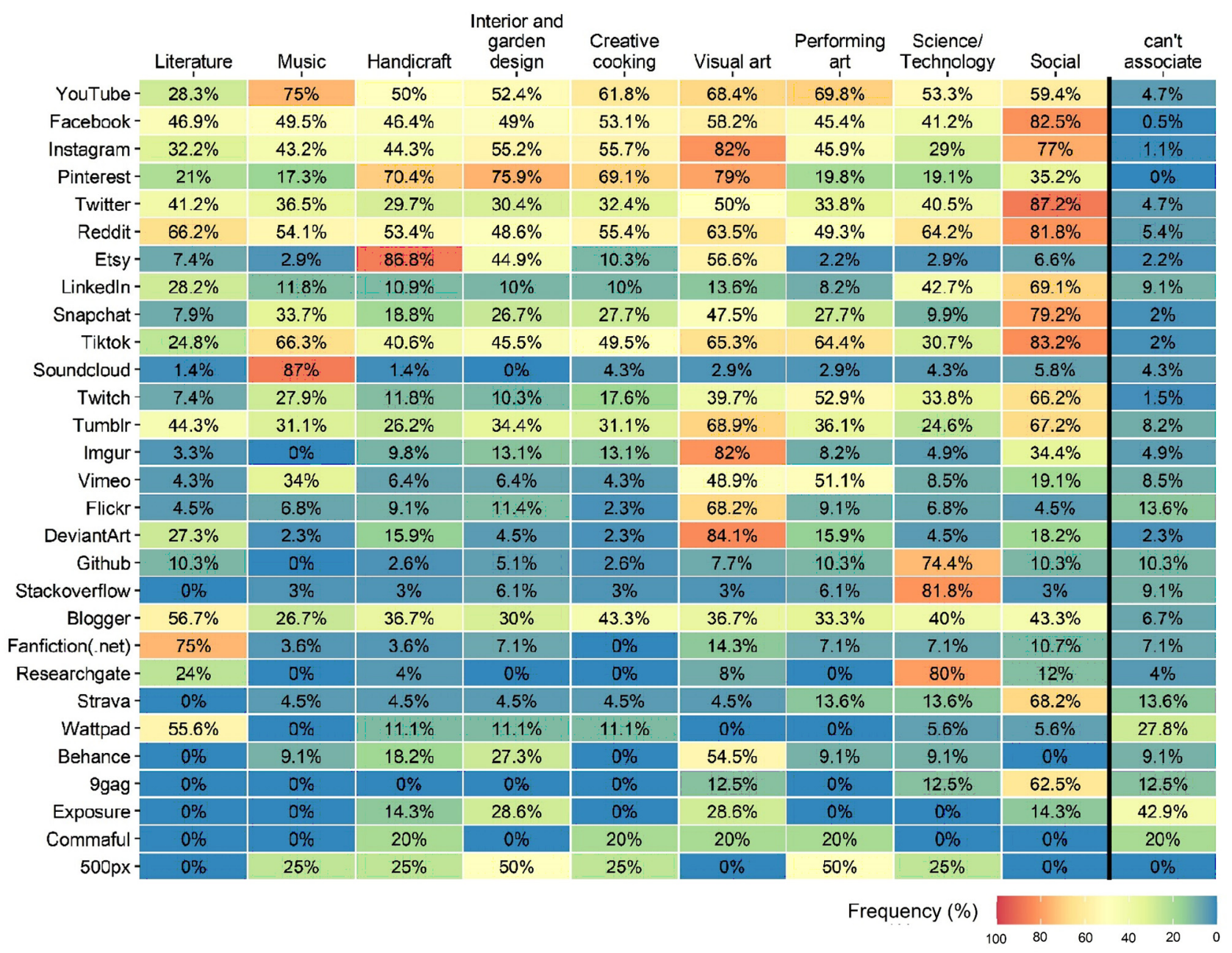

Figure 3. Associations between online platforms and creative domains

Notes. Percentage scores for each platform based on the users (see Figure 2). Participants could choose multiple domains.

Looking at each creative domain separately, we found that literary creativity was most strongly associated with Fanfiction.net; (75\%). Musical creativity was most strongly associated with Soundcloud (87\%). For the remaining domains, the strongest links are as follows: Handicraft: Etsy (86.8\%); Interior and garden design: Pinterest (75.9\%); Creative cooking: Pinterest (69.1\%); Visual art: DeviantArt (84.1\%); Performing art: YouTube (69.8\%); Science/Technology: Stackoverflow (81.8\%); Social creativity: Twitter (87.2\%). Hence, creative domains clearly differ in their preferred outlets.

Why do I post. In a final step, we analyzed the motives for posting creative content online. Participants generally agreed with eleven of the twelve statements (> 50\%), with the strongest motives reflecting the enabling nature of online platforms, allowing users to bring pleasure to others by showing their 
creative skill (Figure 4). They only tended to disagree that monetizing was an important motive for posting their creative work. Participants further named sixteen additional motives, most of which were largely similar to the 12 original motives (e.g., "Engage with the community of my niche." is strongly reflected in the statement "I want to engage with others in discussing my work."). A few users also added motives such as "fun" to the list, suggesting that the effort put into showcasing one's creative work may indeed serve a purpose in itself. One motive, however, that was reasonably distinct was the opportunity to post creative content online for reasons of storage (i.e., archiving). Arguably, this motive may not be specifically related to posting creative content online but rather to posting content of all kinds online. The same holds true for "keeping one's family updated" - a behavior that will almost certainly go beyond posting creative content online.

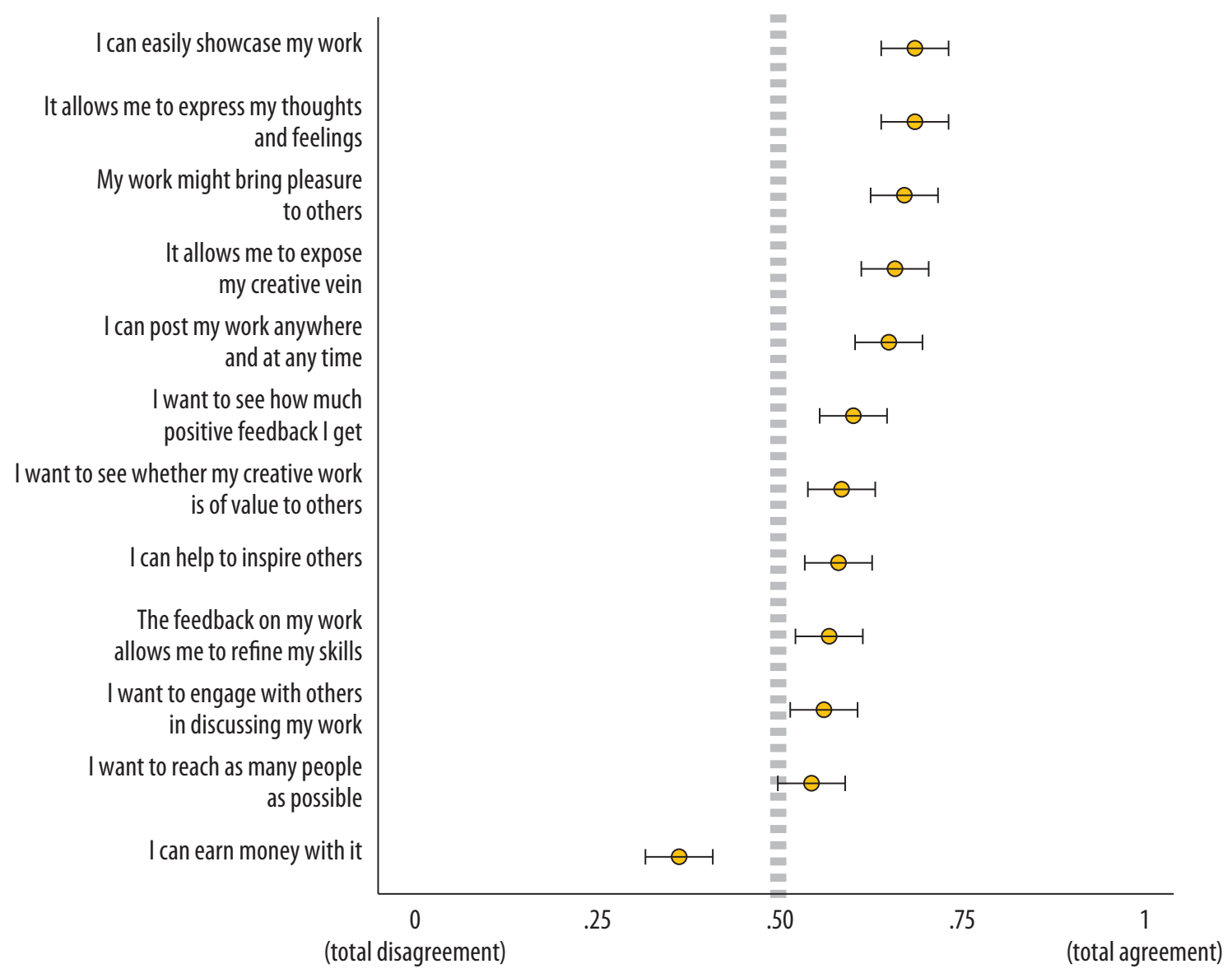

Figure 4. Motives for posting creative content online, sorted by extent of agreement. Error bars reflect SEM $(95 \% \mathrm{Cl})$

\section{DISCUSSION}

The main goal of the present study was to explore what, where, and why creative work is shared online to inform future research on creativity in online spaces. In general, we found that most people regularly post their own creative content online, with more than $90 \%$ reporting to do so at 
least a few times per year. Considering that the sample reflected a broad age range, this posting activity may be taken to corroborate what has been called a "digital creativity boom" (Literat \& Glăveanu, 2018).

Online posting activity of creative content was especially frequent in the domains of creative cooking, literature, and visual art. For example, some reported posting their vegetarian and vegan recipes; one participant even reported uploaded recipes that meet the needs of people with intolerances. Others shared their poems, short stories, and book reviews online. Visual art postings ranged from wildlife photography, over time-lapse shootings to instructional videos on woodworking. In contrast, creative works in the domains of performing arts and music were shared least frequently, which may point to the higher effort associated with creating products in these domains. Interestingly, the prevalence of creative domains in online posting is similar to that previously observed for actual creative behaviors (Benedek et al., 2020). Everyday creative behaviors are also highly frequent in the domains of creative cooking and visual arts and relatively infrequent in performing arts, suggesting that posting of creative content reflects to some extent the frequency of own creative behaviors. Nevertheless, there were also notable differences, as creative behaviors more commonly than postings referred to interior and garden design and less commonly to the literature domain. These differences indicate that the frequency of posting of own creative products may be moderated by how amenable these products are for sharing online.

Considering the creative domains together with the verbatim descriptions of the shared creative content revealed that the creative domain may not only refer to the actual offline behavior but also to the way of how it is presented online. As shown in the previous example (creating instructional videos on woodworking), this would actually represent a creative activity in the domain of handicrafts, while creating an entertaining video about the process would involve further creativity related to the domain of visual arts. In other words, depending on how much originality is put into the work of adapting content for effective online presentations (e.g., by means of digital art techniques; Xing, 2018), this process of transformation can represent a creative act in itself. This becomes most apparent when the topic of the online post is not creative in itself but only potentially the way it is presented (e.g., creating original unboxing videos). The consideration of both the levels of generation and presentation complicates the clear allocation of posted creative content to established creativity domains. We thus need to acknowledge that online representations of creative work potentially involve separable creative acts - that of creating the work in the first place and that of crafting an appealing online appearance - each of which may involve skills related to different creative domains.

It was surprising that $37.6 \%$ of all participants reported at least sometimes posting creative content from the social domain. This could be partly due to the omnipresence of the term social media, which may have led users to associate social networking sites such as Linkedln with social creativity, independent of whether they actually seek or post creative content from the social domain. While we can only speculate in that regard, it becomes increasingly hard to disentangle social interaction and (social) creativity in online spaces, considering it as a sociocultural act (Glăveanu, 
2015). As an example, one may consider blogging and vlogging as forms of social creativity, given their orientation towards readers or viewers, while others would consider them to reflect creativity in literature, and visual art, respectively, considering the way it was produced, and yet others may view blogging and vlogging to be creative activity in the domain of the featured content (e.g., blogging about dance concerns the creative domain of performing art). Newly emerging forms of content (Literat, 2019) and new forms of participation (Literat \& Glăveanu, 2016), further complicate the issue. For example, for an increasing number of videogames, developers enable users to create new or adapt existing content of their games that can then be downloaded by other users and implemented into their game (e.g., Steam workshop). These novel types of creative online activity are not easily located in established creative domains and thus call for a multi-domain and process perspective (Benedek et al., 2019). Moreover, it raises the interesting conceptual question of whether such native forms of online creative activities may be even considered to constitute an independent domain of online creativity.

Looking at usage patterns, we found that participants commonly use platforms to view creative work by others. This highlights the function of online platforms as an immense resource of creative content that can be delved into to find pleasure and inspiration. It seems evident that viewing creative content happens much more frequently than posting one's own content, as the latter takes more effort, but we identified two platforms with exceptionally high posting rates: Facebook and Instagram had by far the highest posting usage rates (40-50\%), with Instagram being even used more frequently for posting than for viewing. The high posting rates for these platforms may be partly due to the fact that they facilitate easy sharing in many formats (i.e., postings, stories, reels, live-streaming) and provide professional editing tools (e.g., filters), which makes it easy and fun to create appealing postings. In general, posting something online often requires just a few clicks, but it can also be done in very thoughtful, elaborated ways. As an example, Instagram postings can be seen as little mosaics that, when viewed in total (i.e., looking at the list of postings in one's profile), result in a coherent picture. This again highlights that posting can become a creative act in itself. To give another example, posting a time-lapse recording of a drawing process may offer one's followers a new perspective on the production of creative content, and can simultaneously be visually and even emotionally appealing, considering the option to underline the video with a fitting tune that conveys an artists' feelings during the act of drawing. While this study did not assess the peculiarities of creative postings in that regard, it suggests that providing users with different tools to showcase, polish, and augment their content clearly encourages creativity in the process of online sharing.

Analyses showed that most creativity domains have dedicated platforms. Literature was most associated with Fanfiction.net, music with Soundcloud, handicraft with Etsy, and interior and garden design with Pinterest. Other creative domains were strongly associated with several platforms: science and technology was associated with Github, Stackoverflow, and ResearchGate, performing art was associated with YouTube and TikTok, and visual arts with Instagram, Imgur, DeviantArt, and Pinterest. Yet, a greater number of platforms were connected to social creativity, including Twitter 
and Tiktok. The weakest associations were found for creative cooking (Pinterest) and performing art (YouTube), suggesting that creative work from these domains is not represented at a few flagship platforms but rather at more diverse online spaces. A closer look also revealed that some platforms, especially social media platforms, are relevant for many creative domains. This demonstrates how social media are "teeming with the products of everyday digital creativity" (Literat, 2019, p. 1169). For example, YouTube was highly popular among our sample and associated with a broad range of creative content across all creative domains. Another example is the online platform Blogger, which was associated with creative content of all domains in a very balanced way. While blogging strongly relies on writing, it does not limit users on what they write about. Thus, these versatile platforms can be considered as domain-general, in equally featuring all creative domains, yet potentially focusing on the preferred presentation medium (e.g., video for YouTube, versus text and pictures for Blogger). In contrast, other platforms were clearly domain-specific in being associated with only one creative domain. These platforms often regulate the type of content (e.g., Flickr only presents photography) and at the same time offer several features tailored to this content (e.g., Flickr provides all relevant technical information with each photo, such as shutter speed). This domain-specific focus also explains why these platforms usually attract a smaller and more specialized audience.

Our selection of platforms seems to have covered many popular sites, but of course, it was by no means exhaustive. Aiming for an English-speaking sample, we did not include platforms that are popular in eastern parts of the globe or focus on a specific language like many literature platforms (e.g., the German platform Story.one). The survey further revealed relevant outlets of creativity that were not considered in the first selection, such as Thingiverse (a platform where people can upload and browse through a variety of digitalized models for 3D-printable objects). On the other hand, we included Snapchat in our list of platforms, although it is essentially a smartphone app and - to our knowledge - cannot be fully accessed through a website. This was done as we figured that Snapchat provides users with a significant portion of creativity-enabling tools to share visual content in creative ways. To be sure, there are many more relevant platforms, many of which serve smaller communities of highly specialized creative users (e.g., Ravelry, a platform for knitting, crocheting, and fiber art). Furthermore, as this is a very dynamic field, new online platforms and apps can constantly be expected to emerge (e.g., Clubhouse).

This research also explored the reasons for sharing own creative work online. People especially endorsed statements reflecting an appreciation of the technological benefits of the Internet, as platforms are considered to facilitate the exhibition of creative work. Thus, they feel that they can transport their emotions and feelings on the go via their creative products and bring pleasure to others - who, in turn, reward them with positive feedback. However, the majority of users disagreed with the statement "I can earn money with it", suggesting that they do not view their postings of creative content in their leisure time as a source of potential income. This motive will certainly be stronger among creative professionals and with commercial online platforms (e.g., iStockphoto), which were not the focus of this study. Future research may build upon these findings and consider more platform-specific motives to unravel motivational differences for creative action on various 
online platforms and how they may be moderated by individual differences such as in expertise and commercial interests. Two additional motives that were contributed by the participants and should be considered for future studies are archiving one's creative work via posting it and the enjoyment of the creative act of posting.

The present study provides an overview of creative behavior on online platforms and gained first insight into platform-specifics and underlying motives for posting creative content. It revealed the high prevalence of sharing and viewing creative content online, highlighting the important role of the Internet as a showcase of creative activity and as a place to find creative inspiration. Findings further suggest that the online world must not just be viewed as an outlet for offline creative behaviors but actively encourages creative behavior by offering unique ways to present content in original and effective ways in online environments. Importantly, the online world has even coined entirely new types of creative behaviors and products (e.g., memes) that are hard to accommodate in traditional classifications of creative domains. We have still identified some of the factors that shape creativity in online outlets, such as what platforms are popular for viewing versus posting content and what are domain-specific versus domain-general. Future research may continue from here to investigate what makes some online platforms more popular than others for sharing creative work or, at the individual level, find out why some users are more successful in gaining attention for their creative work than others. Here, it would be interesting to see how metrics such as likes or karma reflect the creativity of postings and how online feedback influences future creative work.

As digitalization pervades all areas of life, this equally applies to creative behavior. We already see an enormous range of creative expression in the online worlds, and this is expected to further increase with time, and can even become temporarily fueled by global crises such as the COVID19 pandemic (Karwowski et al., 2021; Lopez-Persem et al., n.d.). Future research is challenged to understand how online environments facilitate or affect traditional forms of creative behavior, as well as to keep track of entirely new online manifestations of creativity. After all, the high availability of shared creative work online does not only benefit dedicated audiences but also interested creativity researchers.

\section{References}

Bakhshi, S., \& Gilbert, E. (2015). Red, purple and pink: The colors of diffusion on pinterest. PLoS ONE, 10(2), 1-20. https://doi.org/10.1371/journal.pone.0117148

Benedek, M., Bruckdorfer, R., \& Jauk, E. (2020). Motives for Creativity: Exploring the What and Why of Everyday Creativity. Journal of Creative Behavior, 54(3), 610-625. https://doi.org/10.1002/jocb.396

Benedek, M., Christensen, A.P., Fink, A., \& Beaty, R.E. (2019). Creativity assessment in neuroscience research. Psychology of Aesthetics, Creativity, and the Arts, 13(2), 218-226. https://doi.org/10.1037/aca0000215

Brabham, D.C. (2010). Moving the crowd at threadless: Motivations for participation in a crowdsourcing application. Information Communication and Society, 13(8), 1122-1145. https://doi.org/10.1080/136911 81003624090

Bruns, A. (2008). Blogs, Wikipedia, Second Life, and Beyond: From Production to Produsage. Peter Lang. 
Chae, J. (2018). Reexamining the relationship between social media and happiness: The effects of various social media platforms on reconceptualized happiness. Telematics and Informatics, 35(6), 1656-1664. https://doi.org/10.1016/j.tele.2018.04.011

de Saint Laurent, C., Glăveanu, V., \& Chaudet, C. (2020). Malevolent Creativity and Social Media: Creating Anti-immigration Communities on Twitter. Creativity Research Journal, 32(1), 66-80. https://doi.org/10. 1080/10400419.2020.1712164

Dumas, T.M., Maxwell-Smith, M., Davis, J.P., \& Giulietti, P.A. (2017). Lying or longing for likes? Narcissism, peer belonging, loneliness and normative versus deceptive like-seeking on Instagram in emerging adulthood. Computers in Human Behavior, 71, 1-10. https://doi.org/10.1016/j.chb.2017.01.037

Glăveanu, V. (2015). Creativity as a Sociocultural Act. Journal of Creative Behavior, 49(3), 165-180. https://doi. org/10.1002/jocb.94

Helmond, A. (2015). The Platformization of the Web: Making Web Data Platform Ready. Social Media + Society, 1-11. https://doi.org/10.1177/2056305115603080

Hennessey, B.A. (2019). Motivation and Creativity. In J.C. Kaufman \& R.J. Sternberg (Eds.), The Cambridge Handbook of Creativity (2nd ed., pp. 374-395). Cambridge University Press. https://doi.org/10.1017/978 1316979839

Kim, N.W. (2017). Creative Community Demystified: A Statistical Overview of Behance. https://www.researchgate.net/publication/314182350_Creative_Community_Demystified_A_Statistical_Overview_of_Behance

Kircaburun, K., Griffiths, M.D., Şahin, F., Bahtiyar, M., Atmaca, T., \& Tosuntaş, Ş.B. (2020). The Mediating Role of Self/Everyday Creativity and Depression on the Relationship Between Creative Personality Traits and Problematic Social Media Use Among Emerging Adults. International Journal of Mental Health and Addiction, 18(1), 77-88. https://doi.org/10.1007/s11469-018-9938-0

Literat, I. (2012). The Work of Art in the Age of Mediated Participation. Internation Journal of Communication, 11, 2962-2984. http://ijoc.org/index.php/ijoc/article/view/1531

Literat, I. (2015). Crowdsourced Art: Activating Creative Participation in Online Spaces. University of Southern California.

Literat, I. (2019). Make, share, review, remix: Unpacking the impact of the internet on contemporary creativity. Convergence, 25(5-6), 1168-1184. https://doi.org/10.1177/1354856517751391

Literat, I., \& Glăveanu, V. (2018). Distributed Creativity on the Internet: A Theoretical Foundation for Online Creative Participation. International Journal of Communication, 12(0), 16. https://ijoc.org/index.php/ ijoc/article/view/7621

Literat, I., \& Glăveanu, V.P. (2016). Same but Different? Distributed Creativity in the Internet Age. Creativity. Theories - Research - Applications, 3(2), 330-342. https://doi.org/10.1515/ctra-2016-0020

Literat, I., \& van den Berg, S. (2019). Buy memes low, sell memes high: vernacular criticism and collective negotiations of value on Reddit's MemeEconomy. Information Communication and Society, 22(2), 232249. https://doi.org/10.1080/1369118X.2017.1366540

Lopez-Persem, A., Bieth, T., Guiet, S., \& Ovando-tellez, M. (n.d.). Through thick and thin: changes in creativity during the first lockdown of the Covid-19 pandemic. https://doi.org/10.31234/osf.io/26qde

Mull, I.R., \& Lee, S.E. (2014). "PIN" pointing the motivational dimensions behind Pinterest. Computers in Human Behavior, 33, 192-200. https://doi.org/10.1016/j.chb.2014.01.011

Richterich, A. (2014). 'Karma, Precious Karma!' Karmawhoring on Reddit and the Front Page's Econometrisation. Journal of Peer Production, 4(1), 1-12. http://peerproduction.net

Runco, M. (2015). The Real Creativity Crisis. Creativity \& Human Development, Jan, 1-10.

Seiffert-Brockmann, J., Diehl, T., \& Dobusch, L. (2018). Memes as games: The evolution of a digital discourse online. New Media \& Society, 20(8), 2862-2879. https://doi.org/10.1177/1461444817735334

Sheldon, P., \& Bryant, K. (2016). Instagram: Motives for its use and relationship to narcissism and contextual age. Computers in Human Behavior, 58, 89-97. https://doi.org/10.1016/j.chb.2015.12.059 
Vásquez, C., \& Aslan, E. (2021). "Cats be outside, how about meow": Multimodal humor and creativity in an internet meme. Journal of Pragmatics, 171, 101-117. https://doi.org/10.1016/j.pragma.2020.10.006

Verduyn, P., Ybarra, O., Résibois, M., Jonides, J., \& Kross, E. (2017). Do Social Network Sites Enhance or Undermine Subjective Well-Being? A Critical Review. Social Issues and Policy Review, 11(1), 274-302. https:// doi.org/10.1111/sipr.12033

Xing, B. (2018). Creativity and Artificial Intelligence: A Digital Art Perspective. Social Science Research Network, 1-55.

YouTube Official Blog. (2021). Youtube By The Numbers. blog.youtube.com/press

Zittrain, J.L. (2014). Reflections on Internet Culture. Journal of Visual Culture, 13(3), 388-394. https://doi.org/1 $0.1177 / 1470412914544540$ 